\title{
Global, cancer-specific microRNA cluster hypomethylation was functionally associated with the development of non-B non-C hepatocellular carcinoma
}

Masanori Nojima', Takeshi Matsui ${ }^{2,3}$, Akihiro Tamori ${ }^{4}$, Shoji Kubo ${ }^{5}$, Ken Shirabe ${ }^{6}$, Koichi Kimura $^{6}$, Mitsuo Shimada ${ }^{7}$, Tohru Utsunomiya ${ }^{7}$, Yasuteru Kondo ${ }^{8}$, Etsuko lio $^{3}$, Yutaka Naito ${ }^{9}$, Takahiro Ochiya ${ }^{9}$ and Yasuhito Tanaka ${ }^{3 *}$

\begin{abstract}
Background: While hepatitis B and C viral infection have been suppressed, non-B non-C hepatocellular carcinoma (NBNC-HCC) is considered to be rising in incidence terms in some developed countries where prevalence of those viral infections among HCC patients had been very high (such as Japan, Korea, and Italy). To elucidate critical molecular changes in NBNC-HCC, we integrated three large datasets relating to comprehensive array-based analysis of genome-wide DNA methylation ( $N=43$ pairs) and mRNA/miRNA expression ( $N=15$, and 24 pairs, respectively) via statistical modeling.

Results: Hierarchical clustering of DNA methylation in miRNA coding regions clearly distinguished NBNC-HCC tissue samples from relevant background tissues, revealing a remarkable tumor-specific hypomethylation cluster. In addition, miRNA clusters were extremely hypomethylated in tumor samples (median methylation change for non-clustered miRNAs: -2.3\%, clustered miRNAs: -24.6\%). The proportion of CpGs hypomethylated in more than 90\% of the samples was $55.9 \%$ of all CpGs within miRNA clusters, and the peak methylation level was drastically shifted from $84 \%$ to $39 \%$. Following statistical adjustment, the difference in methylation levels within miRNA coding regions was positively associated with their expression change. Receiver operating characteristic (ROC) analysis revealed a great discriminatory ability in respect to cluster-miRNA methylation. Moreover, miRNA methylation change was negatively correlated with corresponding target gene expression amongst conserved and highly matched miRNA sites.

Conclusions: We observed a drastic negative shift of methylation levels in miRNA cluster regions. Changes in methylation status of miRNAs were more indicative of target gene expression and pathological diagnosis than respective miRNA expression changes, suggesting the importance of genome-wide miRNA methylation for tumor development. Our study dynamically summarized global miRNA hypomethylation and its genome-wide scale consequence in NBNC-HCC.
\end{abstract}

Keywords: Global hypomethylation, MicroRNA, MicroRNA cluster, MicroRNA regulation, Statistical modeling, Non-B non-C hepatocellular carcinoma

\footnotetext{
* Correspondence: ytanaka@med.nagoya-cu.ac.jp

${ }^{3}$ Department of Virology and Liver Unit, Nagoya City University Graduate

School of Medical Sciences, Nagoya, Japan

Full list of author information is available at the end of the article
} 


\section{Background}

Hepatocellular carcinoma ( $\mathrm{HCC}$ ) is a common refractory cancer especially in areas where hepatitis virus infection is common, including Japan. (746,000 deaths/782,000 new cases in the world, GLOBOCAN 2012) [1]. While hepatitis $B$ virus (HBV) and $C$ virus (HCV) infection have been suppressed with development of public health measures and medical treatment, non- $\mathrm{B}$ non- $\mathrm{C}$ hepatocellular carcinoma (NBNC-HCC) is rising in incidence terms in Japan [2-4]. The relative proportion of NBNC$\mathrm{HCC}$ in respect to total $\mathrm{HCC}$ has been increasing in etiological terms from approximately $10 \%$ to $15-20 \%$, with elevated rates of nonalcoholic fatty liver disease (NAFLD) also being a potentially contributory factor [2-4]. A similar tendency is observed in some developed countries where prevalence of HBV or HCV infection among HCC patients had been very high (around 80-90\%), such as Korea, and Italy [5]. The mechanism(s) underlying the carcinogenesis of NBNC-HCC can be different from virus-mediated cancers; accordingly, to suppress all types of liver cancer efficiently, the molecular basis of each etiology should be elucidated.

In terms of molecular oncology, altered epigenetic regulation is known to be an important contributory factor in tumor development. Epigenetic marks such as DNA methylation patterns are observed to be significantly different between cancer and non-cancer tissues, and a number of studies have indicated various cancerspecific epigenetic characteristics for every cancer type $[6,7]$. In terms of HCC, altered DNA methylation patterns are also indicative of tumor-associated processes [8-11]. The main focus of DNA methylation research has been on cancer-specific CpG island methylation within promoter regions of individual genes of interest, but the recent development of epigenome-wide analysis approaches has enabled the DNA methylome to be assessed comprehensively $[9,11,12]$. Genome-wide hypomethylation is a common epigenetic change in tumor cells compared to normal cells, as well as regionspecific hypermethylation of promoter regions of cancerrelated genes [13]. Recently, it was reported that HBV encodes a DNA methylation suppressor protein (HBx). This factor is considered oncogenic, with a strong influence on genome-wide methylation experimentally revealed $[12,14,15]$. Whether there are external "epimutagens" in other tumors including $\mathrm{NBNC}-\mathrm{HCC}$ is an important research question.

To study the dynamic interplay between DNA methylation and messenger RNA (mRNA) expression, epigenetic information should be connected to genome-wide expression information; however, such integration is a challenging task. Even if genome-wide surveillance is employed, the full extent of the generated data is not often completely utilized or appreciated. In addition, gene activity is also controlled post-transcriptionally by microRNAs (miRNA), with this complexity making the regulation of general (not local) gene expression more difficult to understand [16]. Oncogenic miRNAs have been reported, as well as oncogenic epigenetic alterations, in the case of HCC $[11,17]$. For example, expression of has-mir-216a and b was found to be upregulated in HCC, with oncogenic behavior also observed for both miRNAs [17]. miRNA expression can also be regulated by DNA methylation, albeit the correlation between these is not always positive or negative and is regiondependent [18]. Moreover, although DNA methylation alterations in $\mathrm{CpG}$ islands within promoter regions have been well studied in the oncology filed, the dynamics and function(s) of DNA methylation in other regions, such as gene bodies or non-island CpG sites, is poorly understood [19].

Here, in this study, to elucidate critical molecular changes in NBNC-HCC, we integrated three large-scale datasets relating to comprehensive analysis of genomewide DNA methylation and mRNA/miRNA expression, together with statistical modeling. We firstly focused on comparison of genome-wide DNA methylome and mRNA transcriptome between tumor and non-tumor background tissues. After detecting tumor-specific differences in the DNA methylome, we explored the consequences of these alterations at a functional level in terms of corresponding expression changes, if present. We also found a remarkably broad pattern of aberrant DNA methylation within miRNA coding regions, with its relationship to target gene expression determined by constructing statistical models. Our finding describes the dynamics of DNA methylation-expression interplay and associated modification by concomitant epigenetic alteration of miRNAs. We believe that this study provides highly generalizable findings relating to global regulation of gene expression as influenced by epigenetic alterations during carcinogenesis.

\section{Results \\ Differences in methylome state between NBNC-HCC tumor tissues and non-tumor background tissues}

Tissues from a total of 43 patients were used for this study, including 13 (30.2\%) females and 30 (69.8\%) males. The mean age of patients was 68.1 years (standard deviation: 12.4). Non-supervised hierarchical clustering ( $1 \%$ randomly extracted) based on genome-wide DNA methylation distinguished NBNC-HCC samples clearly from their background non-tumor tissues (Fig. 1a). Tumor samples were characterized by hypomethylated CpGs (defined as a negative change of methylation level compared to respective background). Hierarchical clustering for the difference in methylation level between paired tumor and background tissues also showed commonly 
a

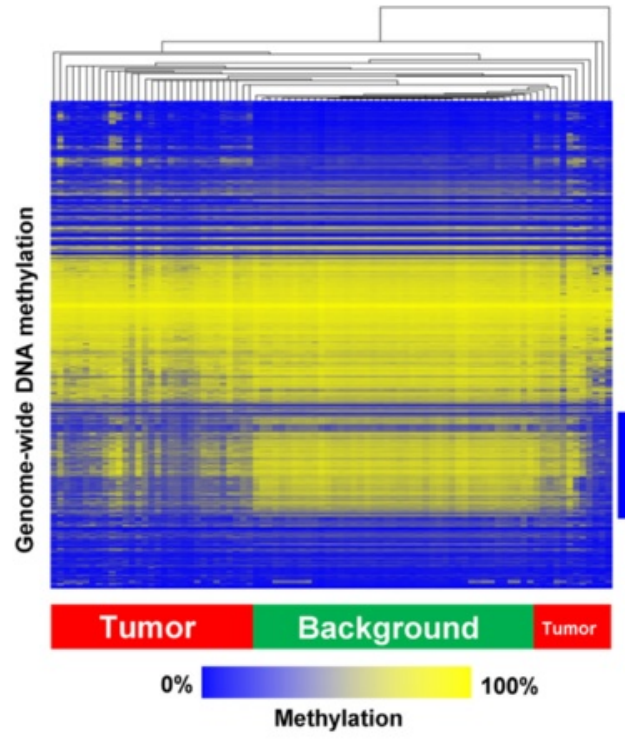

b

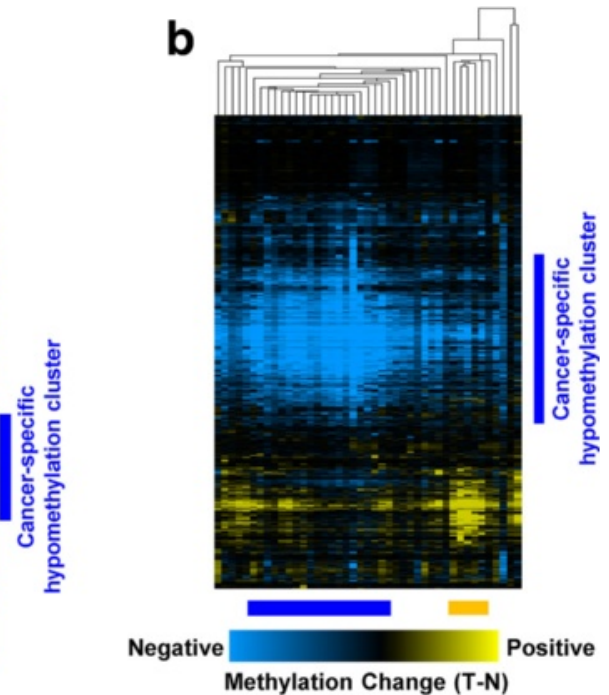

Methylation Change ( $\mathrm{T}-\mathrm{N}$ )

C

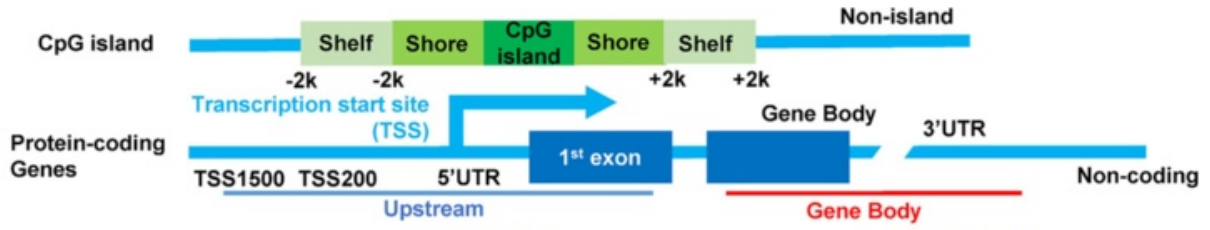

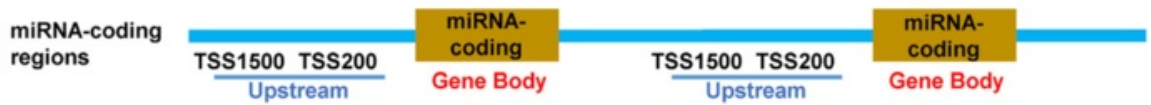

d Upstream

Gene Body

Non-coding

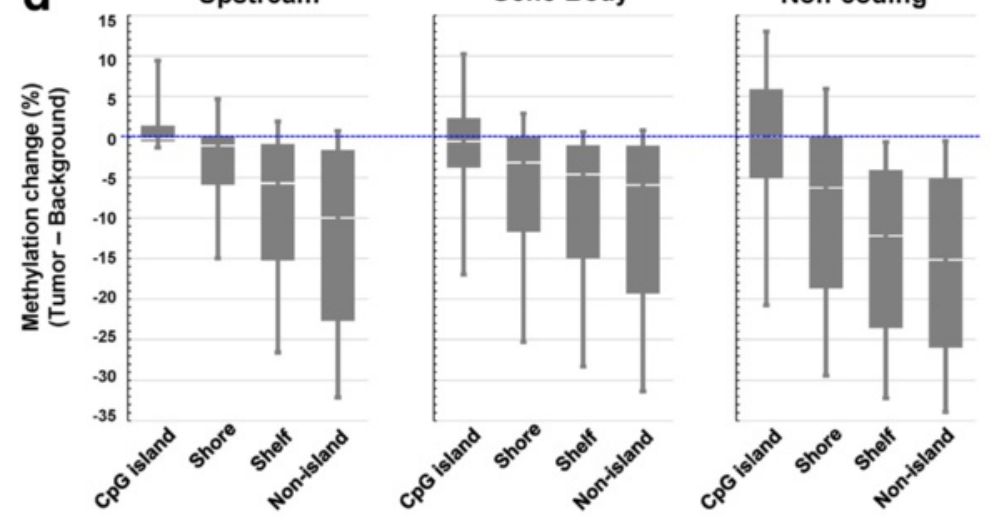

e
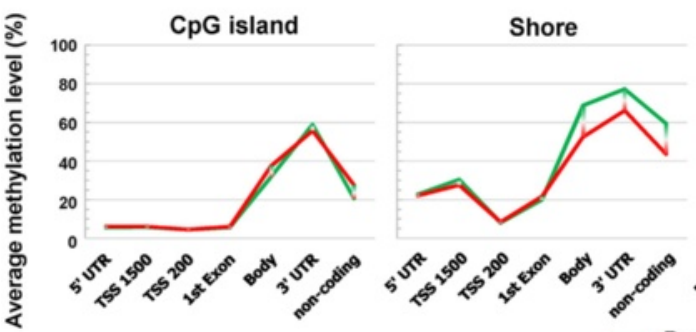

Shelf

Non-island
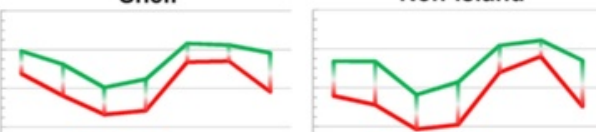

- Background

Fig. 1 (See legend on next page.) 


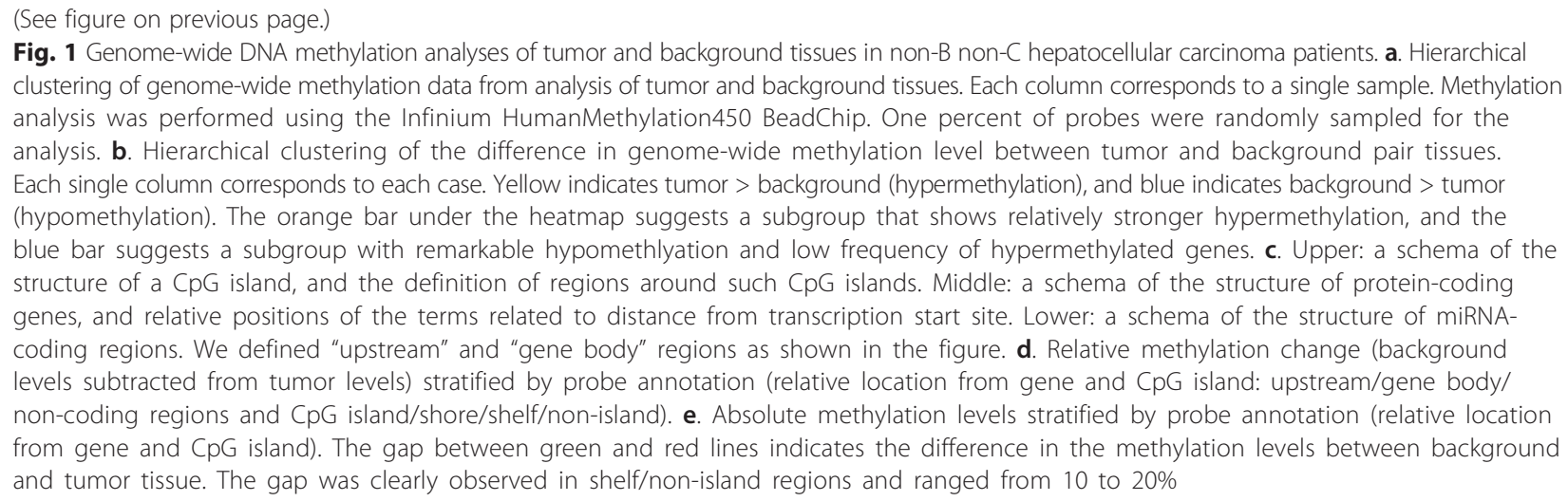

hypomethylated probes in cancer tissues (Fig. 1b). This suggests there are certain subgroups characterized by different methylation patterns (see orange and blue bars in Fig. 1b).

The functional impact of DNA methylation in respect to effects on gene expression differs depending on the characteristics of the respective $\mathrm{CpG}$ locus, such as distance from the transcription start site (TSS) and CpG island. We therefore stratified the data according to these factors based on the annotations defined by the Infinium HumanMethylation450 BeadChip Kit (summarized in Fig. 1c), and plotted the methylation change (tumor - background) in Fig. 1d. Methylation levels were highly downregulated for most sites except CpG islands within upstream regions (where generally promoters exist), where hypermethylation was dominantly observed (see the leftmost bar in Fig. 1d). Medians of the methylation change were distributed from $-15.1 \%$ (non-island in non-coding region) to $0 \%$ (CpG island in any region). Absolute methylation levels in non-tumor background tissues were generally very high in gene body/3'UTR regions, and shelf/non-island regions. Indeed, the methylation levels in background tissues ranged from 70 to $90 \%$, with tumor tissues exhibiting decreases of, on average, 10 to $20 \%$ of this level (Fig. 1e); on the other hand, changes of methylation levels were very discrete within $\mathrm{CpG}$ islands.

\section{Extensive DNA methylation alterations within miRNA coding and clustered miRNA regions}

As described above, hypomethylation was a common observation in tumor tissues. In particular, we detected marked hypomethylation within miRNA coding regions (Fig. 2). As shown in Fig. 2a, clustering on the basis of methylation of miRNA coding regions alone distinguished NBNC-HCC from background tissues, with remarkable evidence of a hypomethylation cluster being found. Methylation levels within miRNA coding regions were highly down-regulated compared to protein-coding gene regions, even when respective upstream regions were compared (Fig. 2b). Moreover, clusters of miRNAs, defined in this study as regions where three or more miRNAs were coded within 15,000 base pairs, were extremely hypomethylated in tumor samples (median for non-cluster miRNAs: $-2.3 \%$, cluster-miRNAs: $-24.6 \%$; Fig. 2c). To investigate this further, the distribution of methylation levels between tumor and background tissues, as well as between non-clustered and clustered miRNAs, were compared (Fig. 2d). As shown in Fig. 2d, the peak methylation level within the clustered miRNAs was clearly shifted from around $84 \%$ to $39 \%$. The sharpness of the peaks and the peak shift was more obvious within clustered miRNAs.

In fact, $66.2 \%$ of the CpG probes within cluster miRNAs were annotated to non-island areas, a highly hypomethylated region, in the tumor. As the proportions of non-island areas were only $36.3 \%$ and $49.7 \%$ within all probes and all miRNAs, respectively, this imbalance can cause a bias. However, using statistical modeling approaches, it was suggested that the methylation level of the clustered miRNAs was significantly lower than that observed with protein-coding genes and non-clustered miRNAs, which was independent of the distance from the TSS and the CpG island (Additional file 1: Table S1, Fig. 2e). In Fig. 2f, we investigated a proportion of significantly hypomethylated CpGs within each miRNA cluster. As a result, $80 \%$ of $\mathrm{CpGs}$ were significantly hypomethylated (paired-t test, $P<0.05$ ) within $56.7 \%$ $(17 / 30)$ of miRNA clusters (17/30, 56.7\%).

\section{Regulation of global miRNA expression by DNA methylation within miRNA coding regions}

We subsequently assessed the association between DNA methylation within miRNA coding regions and expression of respective miRNAs, with baseline level of expression and methylation also taken into consideration. As shown in Additional file 1: Table S2 and Fig. 3a, we constructed and utilized a statistical model (linear mixed 
a

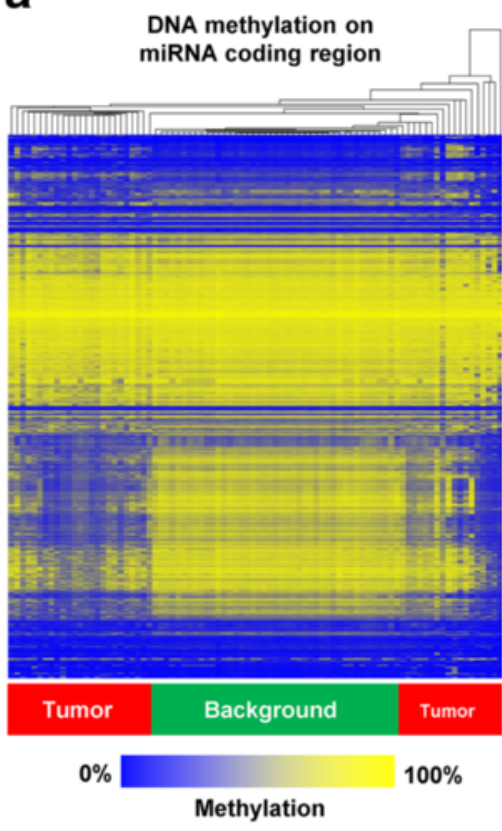

b

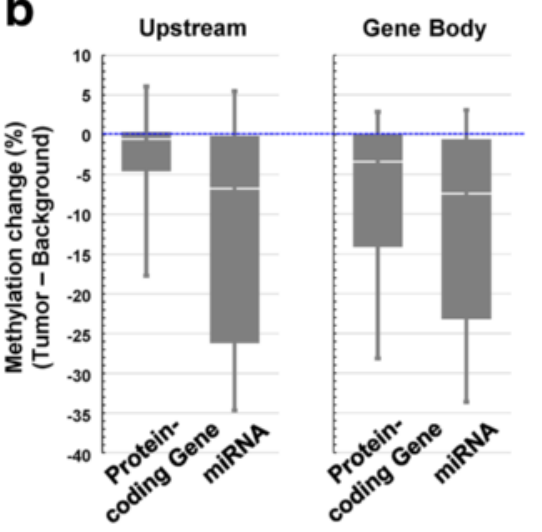

C

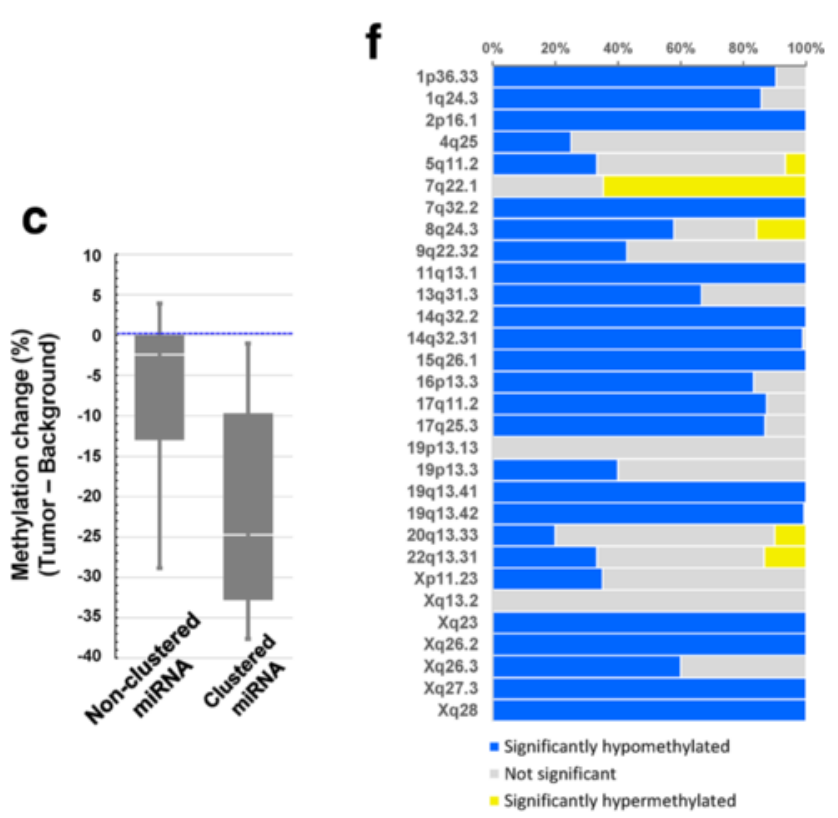

e

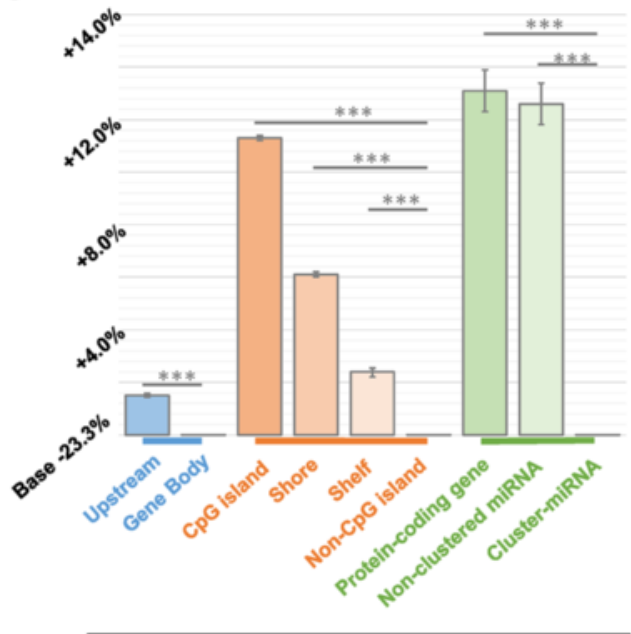

Gene Body + Non-CpG island + Cluster-miRNA $=-23.3 \%+0 \%+0 \%+0 \%=-23.3 \%$

Upstream + CpG island + Coding regions $=-\mathbf{2 3 . 3} \%+1.5 \%+11.3 \%+13.1 \%=+\mathbf{2} .6 \%$

d

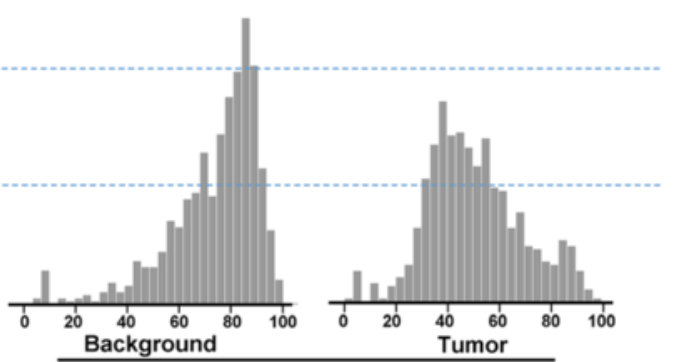
Clustered miRNA methylation

Methylation levels (\%)

Fig. 2 (See legend on next page.) 


\section{(See figure on previous page.)}

Fig. 2 DNA methylation analyses for microRNA coding regions of tumor and background tissues in non-B non-C hepatocellular carcinoma patients. a. Hierarchical clustering of methylation data from tumor and background tissues relating to microRNA-coding regions. Methylation analysis was performed using the Infinium HumanMethylation450 BeadChip. All probes annotated within microRNA regions were used for the analysis. b. Relative methylation change (background levels subtracted from tumor levels) stratified by probe annotations (upstream regions/gene body and protein-coding gene/microRNA). c. Relative methylation change (background levels subtracted from tumor levels) stratified by probe annotations (non-clustered microRNAs/clustered microRNAs). d. Comparison of histogram plots showing methylation levels between background and tumor tissues stratified by non-clustered and clustered microRNA coding regions. e. Visualization of a statistical model showing methylation level in accordance to particular probe annotations (relative location from gene and CpG island, and protein-/miRNA-/clustered miRNA-coding). f. A proportion of significantly hypo/hyper-methylated CpGs within each miRNA cluster

model) for this assessment. The model indicated that DNA methylation changes were generally positively associated with corresponding differences in expression of the respective miRNAs. In particular, following statistical adjustment of miRNA methylation and expression in corresponding background tissues, altered methylation levels of CpG sites in shore, shelf and non-island areas within miRNA coding regions were significantly associated with expression changes of the relevant miRNAs. The expression of conventional protein-coding genes was negatively associated with methylation of $\mathrm{CpG}$ islands within upstream regions; however, this association was not observed in the case of miRNA expression.

\section{Varied, albeit globally positive correlation, between expression and methylation within clustered miRNAs} Almost half of miRNAs are clustered together, which demonstrated impressive hypomethylation in tumor tissues (Fig. 2d). Methylation and expression levels were similarly controlled amongst closely-located miRNAs, with the number of CpG sites involved in transcriptional regulation being relatively small, compared to proteincoding genes (Additional file 2: Figure S1). This suggests that transcriptional regulation via methylation in miRNA coding regions is possibly more visible with cluster-level summarized data. Thus, we then focused on analysis of cluster-level regulation of expression. Summary data regarding methylation and expression levels for miRNA clusters is shown in Additional file 1: Table S3.

As shown in Additional file 3: Figure S2A, methylation and expression levels of the same miRNA cluster were quite similar across all of the background tissues. However, across the tumor tissues, this similarity was disturbed by demethylation tendency within miRNA clusters, with methylation levels in tumor tissues being relatively more varied in nature. A statistical model providing cluster level analysis (constructed in a similar way to that shown in Fig. 3a) also suggested a positive correlation between average change in methylation and expression within each cluster (Additional file 1: Table S4, Fig. 3b). Figure 3c shows in detail the distribution of average miRNA cluster expression according to different methylation levels. Figure 3d also shows the association between differences in methylation and expression of
miRNA clusters, when taking into consideration confounding effects due to background tissue expression. In every stratum stratified by the quartile of background tissue expression, the majority of clusters showed a positive association between methylation and expression change of clustered miRNAs. Pearson's correlation coefficients of their association were $R=0.144$ to 0.211 (not strong, but significant with $P<0.05$ in $3 / 4$ strata). The red circle in the figure indicates up-regulated clusters found within the lowest background expression group, with remarkable up-regulation observed in samples displaying $0 \%$ of difference in methylation (i.e. no methylation change). Even though tumor-specific hypomethylation and up-regulation of miRNA cluster expression were observed within the same miRNA cluster, expression was seemingly paradoxically up-regulated in certain cases within the intact-methylation group. For example, miRNA clusters on 2 p16.1 and 7q32.2 were, respectively, the first and $2^{\text {nd }}$ most up-regulated miRNA clusters across all the samples; both clusters were highly hypomethylated, but tumor-specific upregulation was observed in samples with no methylation change (Fig. 3e).

The detailed methylation changes around the largest cluster on 19q13.42 is shown in Fig. 4a. A remarkable hypomethylation pattern was specifically observed within this miRNA cluster, with methylation differences fading when out of the cluster region. Subsequently, we assessed the extent of correlation between methylation and expression more precisely (at a single probe scale) within several miRNA clusters. Since regulation of the miRNA clusters on $1 \mathrm{p} 36.33$ (the $2^{\text {nd }}$ most downregulated) and $7 \mathrm{q} 32.2$ (the $2^{\text {nd }}$ most up-regulated) have been reported in detail $[20,21]$, we selected these for focused analysis. As shown in Fig. 4b, the methylation levels within miRNA cluster on $1 \mathrm{p} 36.33$ was positively correlated with expression of its linked miRNAs. However, this miRNA cluster also has a large CpG island in the upstream region of the cluster within $20 \mathrm{k}$ bps, with methylation level in this area negatively correlated with expression of miRNAs within the cluster. A similar regulatory pattern was also observed in the case of the miRNA cluster on 7q32.2 (Fig. 4c). The miRNA cluster on 2p16.1, where there is no CpG island within the upstream region of the cluster, demonstrates only a 


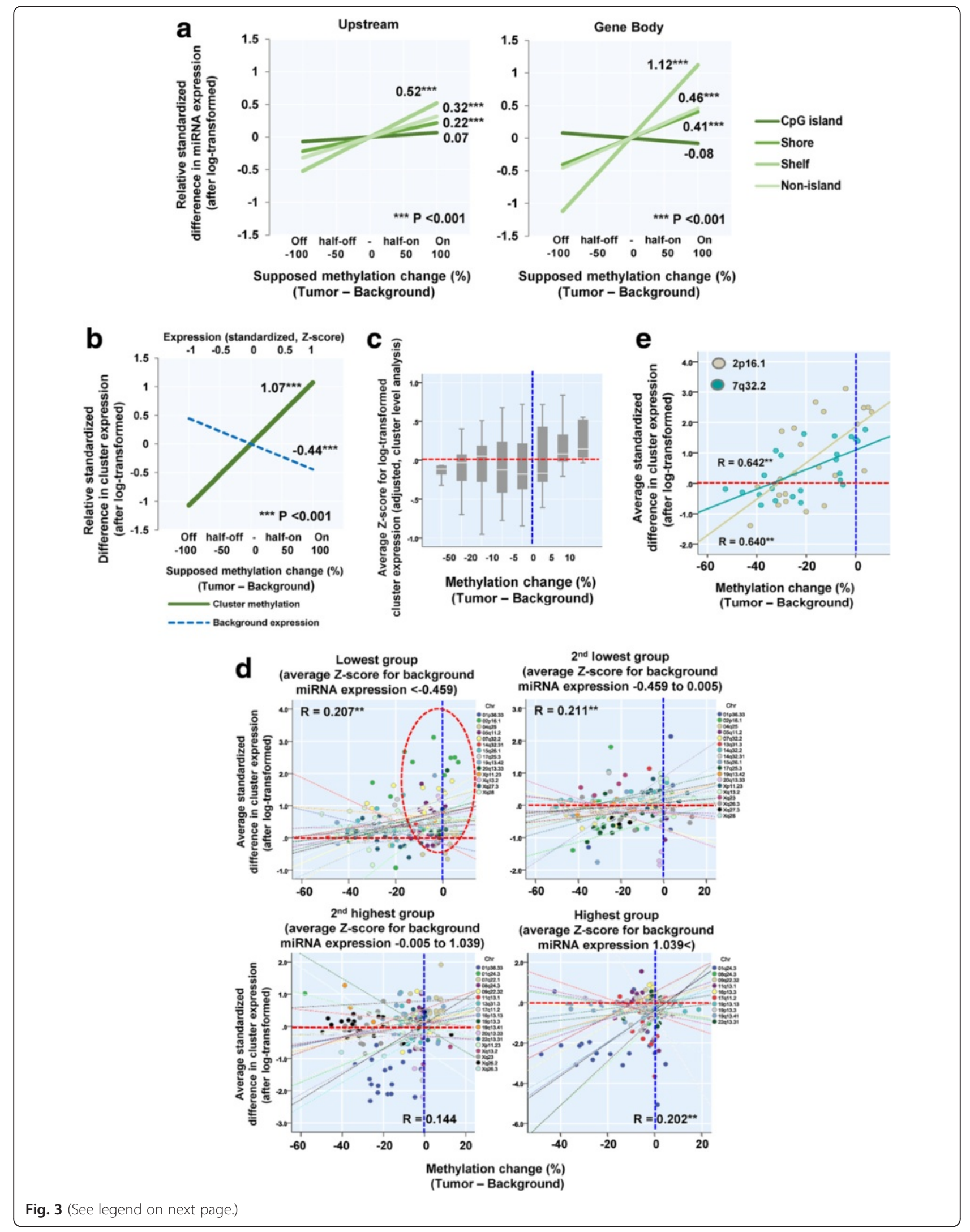




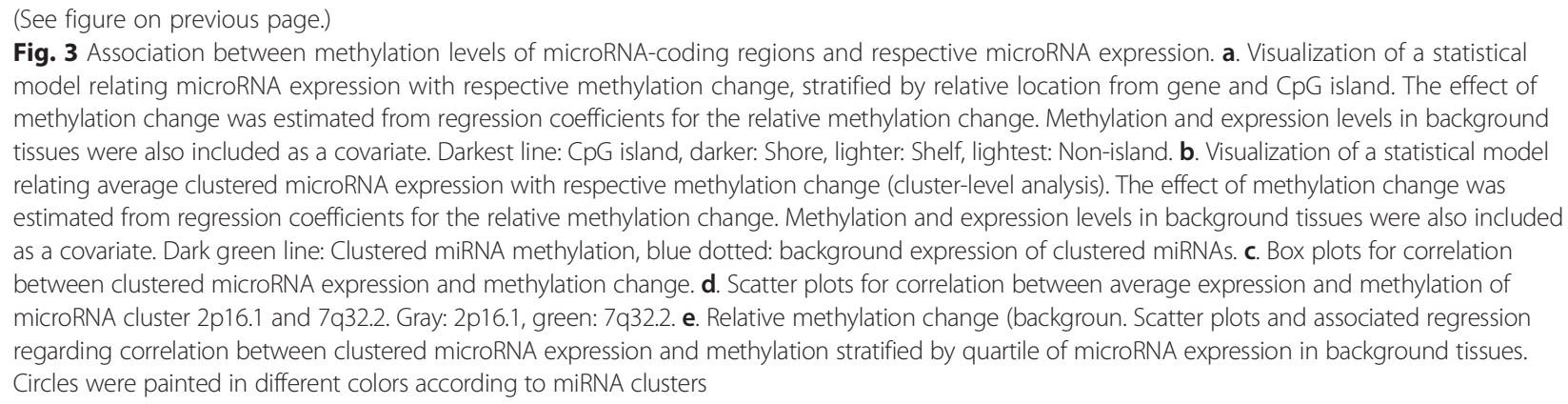

simplistic positive correlation (Fig. 4d). Correlation coefficients determined between methylation and expression for miRNA clusters with upstream sequences within $20 \mathrm{k}$ bps are summarized in Fig. 4e. Consistent with the analysis shown for 2p16.1, methylation at CpG sites in non-island areas was highly positively correlated with expression. CpG islands were mainly located within upstream regions of miRNA clusters (68.8\%), with approximately half of these showing a negative correlation in terms of methylation status with expression, such as that evidenced in Fig. 4b and c. Methylation-expression correlation analysis at a chromosome-wide scale is summarized in Additional file 3: Figure S2B, showing correlation coefficients are varied in nature (positive to negative) depending on location.

\section{Ability for tumor discrimination: comparison of expression and methylation data}

A major perceived impact in respect to methylationdependent influences on tumorigenesis is thought to relate to regulation of gene expression. However, clustering using genome-wide expression data, as shown in Fig. 5a, provided little evidence of tumor versus background tissue discriminatory power, in contrast to the potential power of methylation data to produce a robust separation (as seen in Fig. 1a). Ability to provide tumor versus background discrimination was quantified using ROC analysis for every protein-coding gene and miRNA probe (in terms of both methylation and expression). Accordingly, a great discriminatory ability was shown when data from clustered miRNA methylation was utilized (Fig. 5b). Consistent with the results shown in Fig. 5a, methylation was superior to expression in respect to discriminatory power.

\section{Significance of DNA methylation alteration within miRNA coding regions for target gene expression: a genome-wide integrative analysis}

For comparison purposes, a statistical model for proteincoding genes was then constructed and analysed (Additional file 1: Table S5, Fig. 5c). As previously known, methylation differences in $\mathrm{CpG}$ islands within upstream regions was significantly negatively correlated with relative gene expression (regression coefficient $=-0.91$ : decreasing $-0.91 \times$ SD when $100 \%$ methylation increase, $P<$ 0.001 ), with this correlation pattern gradually changing to positive when progressing further from the $\mathrm{CpG}$ island. On the contrary, methylation within gene body regions positively regulated expression, particularly in CpG-sparse regions (shelf and non-island CpGs). Additionally, we assessed the association between expression change of several DNMTs (DNMT1, DNMT3A, DNMT3B) and average methylation difference of clustered miRNAs. Correlation coefficients for z-transformed log-ratio of DNMTs and methylation difference were 0.135 for DNMT1, 0.375 for DNMT3A, 0.093 for DNMT3B.

Finally, we assessed the significance of dynamic hypomethylation in miRNA coding regions for target gene expression in the context of tumor development. As described above, the ability to provide tumor versus background tissue discrimination was easier to achieve when utilizing the methylation status of miRNA coding regions than expression levels of particular miRNAs. We, therefore, suggest that methylation status is also a better estimator of target gene expression than expression of the respective miRNA. Target genes for each miRNA was determined based on TargetScan 7.0 (www.targetscan.org). For this investigation, we selected all conserved miRNA sites and corresponding target genes with greater context++ score (index for degree of matching: lower than -0.2) [22]. We then used a modeling approach similar to that shown in Fig. 5c, but including changes in miRNA methylation, miRNA expression, and target gene methylation (upstream region and gene body) and their absolute values in background tissues. Also, taking into account the affinity of miRNA sites are dependent on the context++ score, we stratified the analysis according to three different levels of context++ score (results summarized in Fig. 6a and Additional file 1: Table S6). Amongst the highly matched miRNA sites (context++ score $<-0.6$ ), miRNA methylation changes were significantly negatively correlated with corresponding target gene expression change independently from miRNA expression (regression 


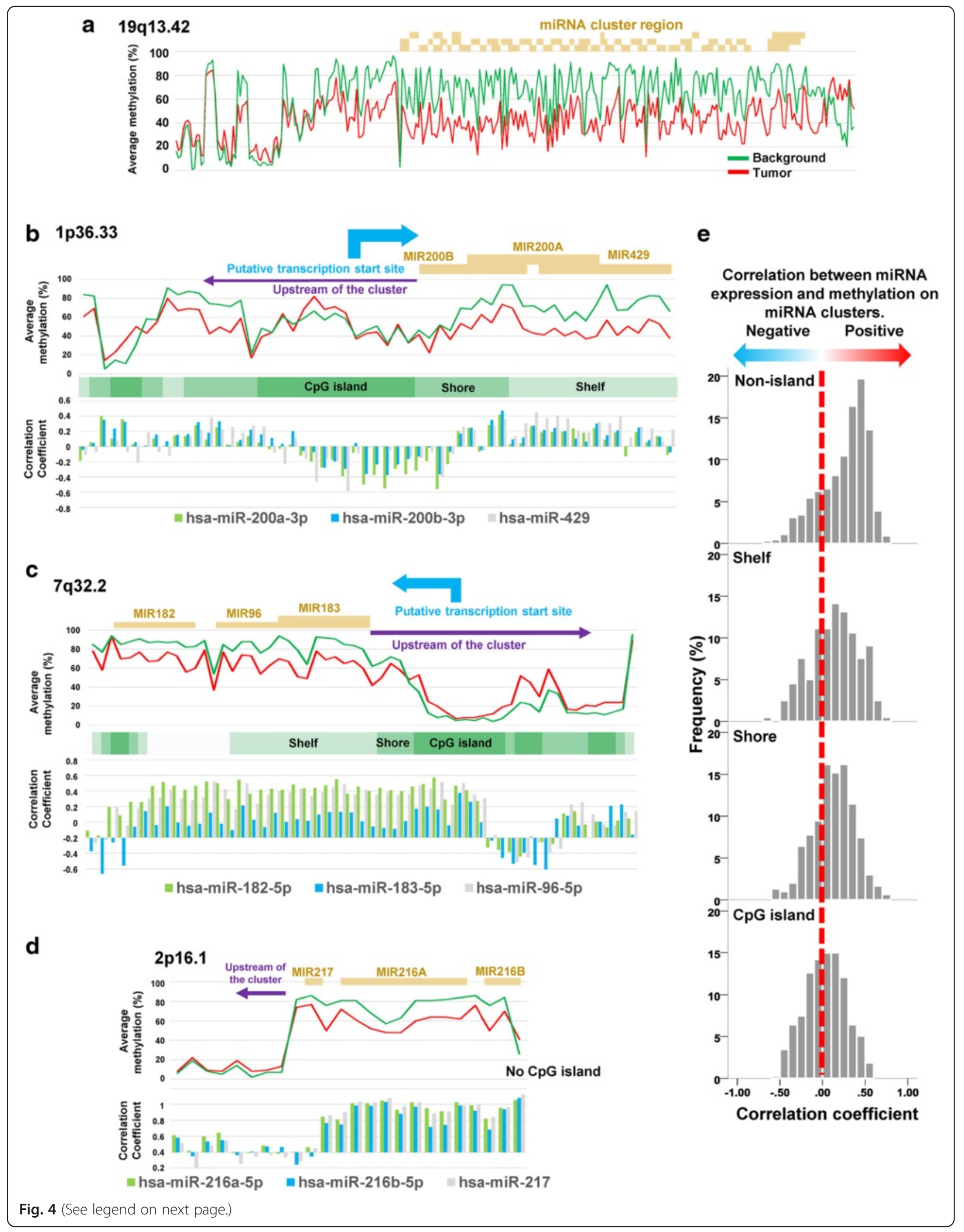


(See figure on previous page.)

Fig. 4 Methylation level for each probe and transcriptional regulation of microRNA clusters. a. Average methylation levels of each probe around the largest microRNA cluster on 19q13.42. Dark yellow bars indicate the miRNA coding regions. $\mathbf{b}$-d. Average methylation levels and correlation coefficients between methylation level and microRNA expression around microRNA cluster on 1p36.33 (B), 7q32.2 (C), and 2p16.1 (D). e. Histogram plots of correlation coefficients between average expression of microRNA clusters and methylation level of miRNA clusters including upstream from clusters within 20k bps stratified by relative location from CpG island

coefficient: $-0.27, P<0.001$; decreasing $-0.27 \times \mathrm{SD}$ in the case of $100 \%$ methylation increase). Absolute value of regression coefficients gradually increased in relation to the context++ score (degree of matching). These data suggest that miRNA hypomethylation is associated with upregulation of target gene expression.

In addition, to identify the influence of the clustered miRNAs, we performed a similar analysis to Fig. 6a only applying the target genes that were targeted by the clustered miRNAs within highly matched miRNA sites (context++ score <-0.6). As shown in Fig. 6b (and Additional file 1: Table S6), we observed the highest regression coefficient for miRNA methylation change than any previous analyses (Regression coefficient: -0.32 , $P<0.001)$. This suggests that the influence of methylation change is more efficient among target genes that are targeted by high-affinity clustered miRNAs. Subsequent hierarchical clustering using such highly-sensitive target genes demonstrated much better separation of tumor from background tissues than in Fig. 5a, showing tumor-specific overexpression of several target genes in which an established oncogene, GPC3, for hepatocellular carcinoma was included (Fig. 6c). A similar analysis to Fig. $5 \mathrm{~b}$ for the same target genes also showed better ability for tumor discrimination (Fig. 6d), compared to the result of non-specified gene expression (see the right upper graph in Fig. 5b). These results suggest an importance of miRNA methylation change for tumor development or progression via regulation of their target gene expression.

\section{Discussion}

Our integrated analyses of methylome and transcriptomic data revealed highly tumor-specific hypomethylation of clustered miRNAs in NBNC-HCCs. The methylation pattern of clustered miRNAs between background and tumor tissues were much more discriminative than expression data from protein-coding genes or miRNAs. The expression change of miRNAs was, on average terms, positively associated with methylation change in the corresponding coding region of miRNA; however, this pattern of regulation was not simple and depended on the relative location from genes. Finally, our result indicated an independent association in respect of miRNA methylation alteration with target gene expression changes from background to tumor tissue. The findings suggest that the broad tumor-specific hypomethylation in miRNA coding regions functionally influence tumor development through regulating target gene expression.

Global hypomethylation is established epigenetic alteration in tumor cells $[14,15]$. Cluster miRNA hypomethylation seems to be happening independently from non-specific global hypomethylation (Fig. 2e). Clustered miRNA hypomethylation has been reported by several studies; however, its significance and relevance to regulation of gene expression is not well understood [23-26]. Jeong $\mathrm{M}$ et.al. reported DNMT3A as being key to clarify and ensure distinctions between low and high DNA methylation areas in the genome [27]. However, in our data, even though DNMT3A expression was highly upregulated, total methylation levels were down-regulated in the tumor samples although a slight positive correlation was observed between global methylation level and expression of each DNMT. The gene encoding the TET protein, which was recently identified to be involved in DNA demethylation [28], was not found as a key factor for tumor-specific hypomethylation in this study. Nevertheless, we could not identify the cause of global hypomethylation observed within NBNC-HCC tissues in this study.

Several studies have reported that hypomethylation of miRNA clusters is associated with re-activation of corresponding miRNA expression [24, 25, 29]. However, we could not conclude that tumor-specific hypomethylation was simply associated with tumor-specific expression (see Additional file 3: Figure S2A and Fig. 3c, d). Paradoxically, up-regulation mostly occurred in methylationintact samples and the expression of hypomethylated samples was not overly up-regulated. It can be explained via the influence of other factors involved in transcription regulation, such as transcription factors, chromosome amplification/deletion, etc. Even if transcriptional signals were turned into "on" through tumor development, if methylation of miRNA coding regions positively regulated affinity downstream of the signal, results as seen for Fig. 3d may be observed.

In addition, as shown in Fig. 4b and c, expression of miRNA clusters on 1 p36.33 and 7q32.2 was negatively correlated with $\mathrm{CpG}$ island methylation within the upstream regions of the clusters, and positively correlated with methylation in miRNA-coding regions. This finding is consistent with previous reports [20, 21]. It indicates that a typical regulation pattern for protein-coding genes 


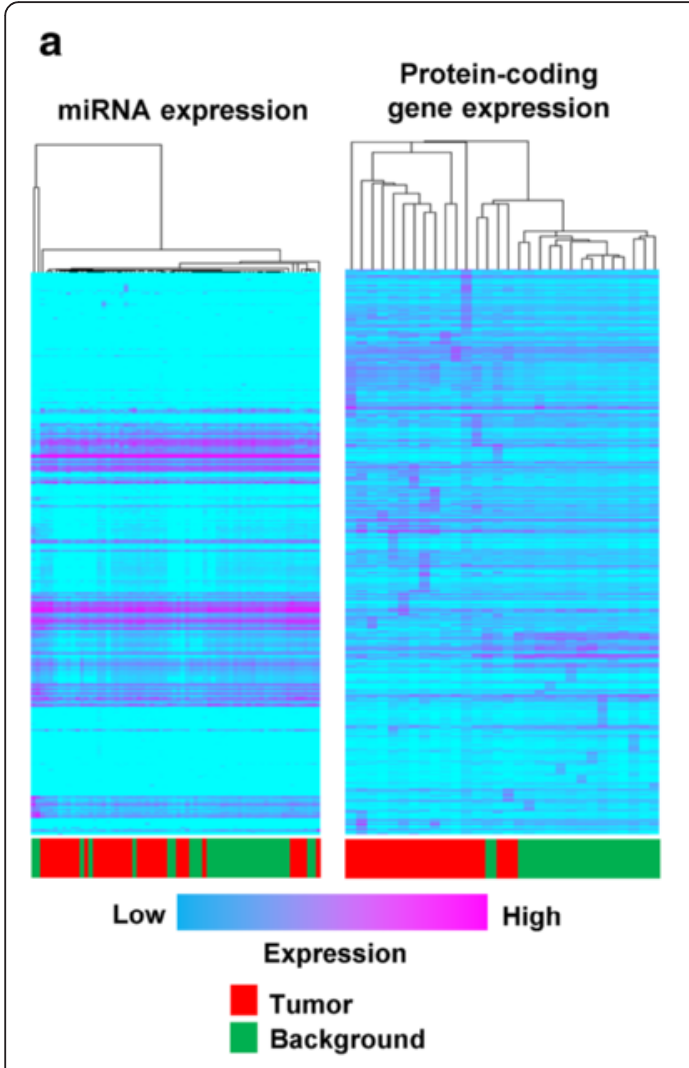

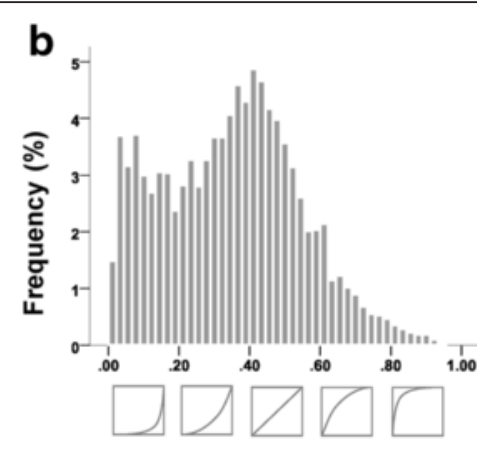

Methylation

(All probes)

SD 0.143

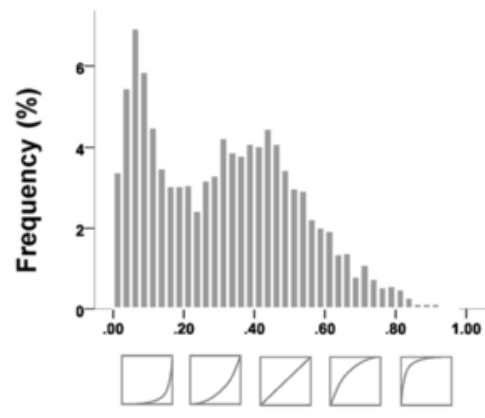

miRNA methylation

Average absolute AUC $=\mathbf{0 . 7 3 4}$ SD 0.153

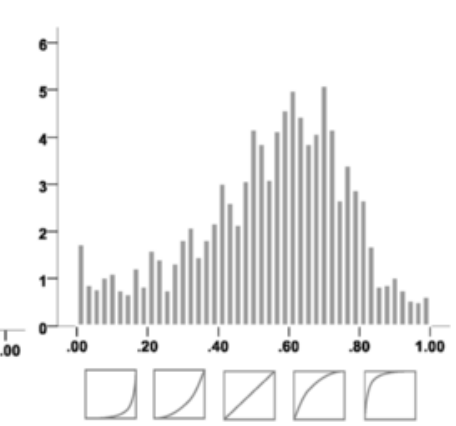

Expression

(All probes)

Average absolute $\mathrm{AUC}=0.686$ SD 0.128

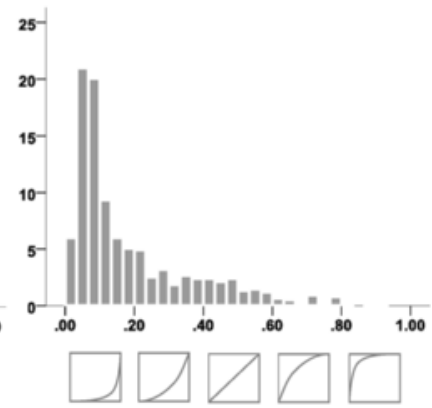

Clustered miRNA methylation Average absolute AUC $=0.832$ SD 0.137

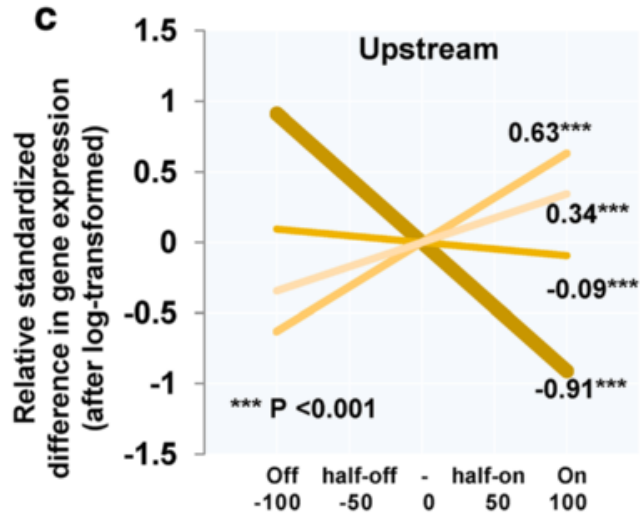

Supposed methylation change (\%) (Tumor - Background)

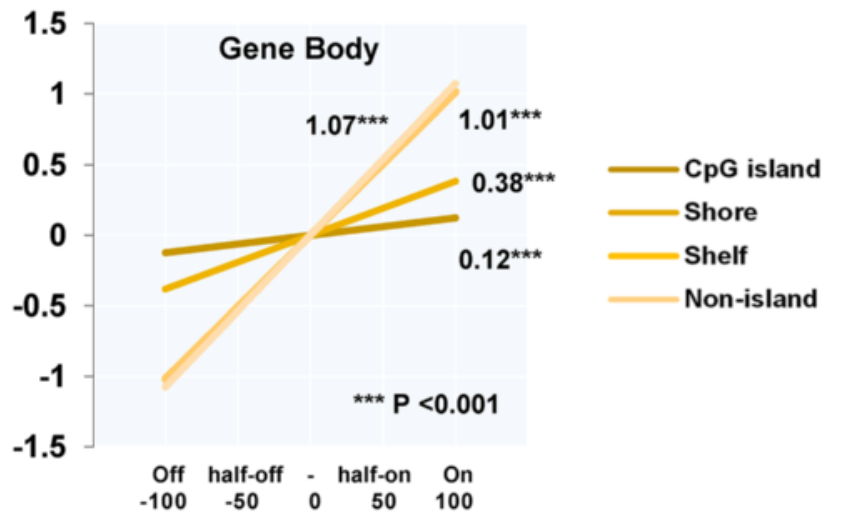

Supposed methylation change (\%) (Tumor - Background)

Fig. 5 Assessment of ability for tumor discrimination, and regulation of protein-coding gene expression by methylation. a. Hierarchical clustering of genome-wide microRNA and protein-coding gene expression analysis data from tumor and background tissues. One percent of probes were randomly sampled for the analysis of protein-coding genes. $\mathbf{b}$. Histogram plots of area under curves (AUC) of repeated receiver operator characteristic (ROC) analysis in respect of ability to discriminate tumor from background tissue. Curves shown below are images of representative results from ROC analysis for various probes. c. Visualization of a statistical model relating expression of protein-coding genes with respective methylation change stratified by relative location from gene and CpG island. The effect of methylation change was estimated from regression coefficients for the relative methylation change. Methylation and expression levels in background were also included as covariate. Darkest line: CpG island, darker: Shore, lighter: Shelf, lightest: Non-island 

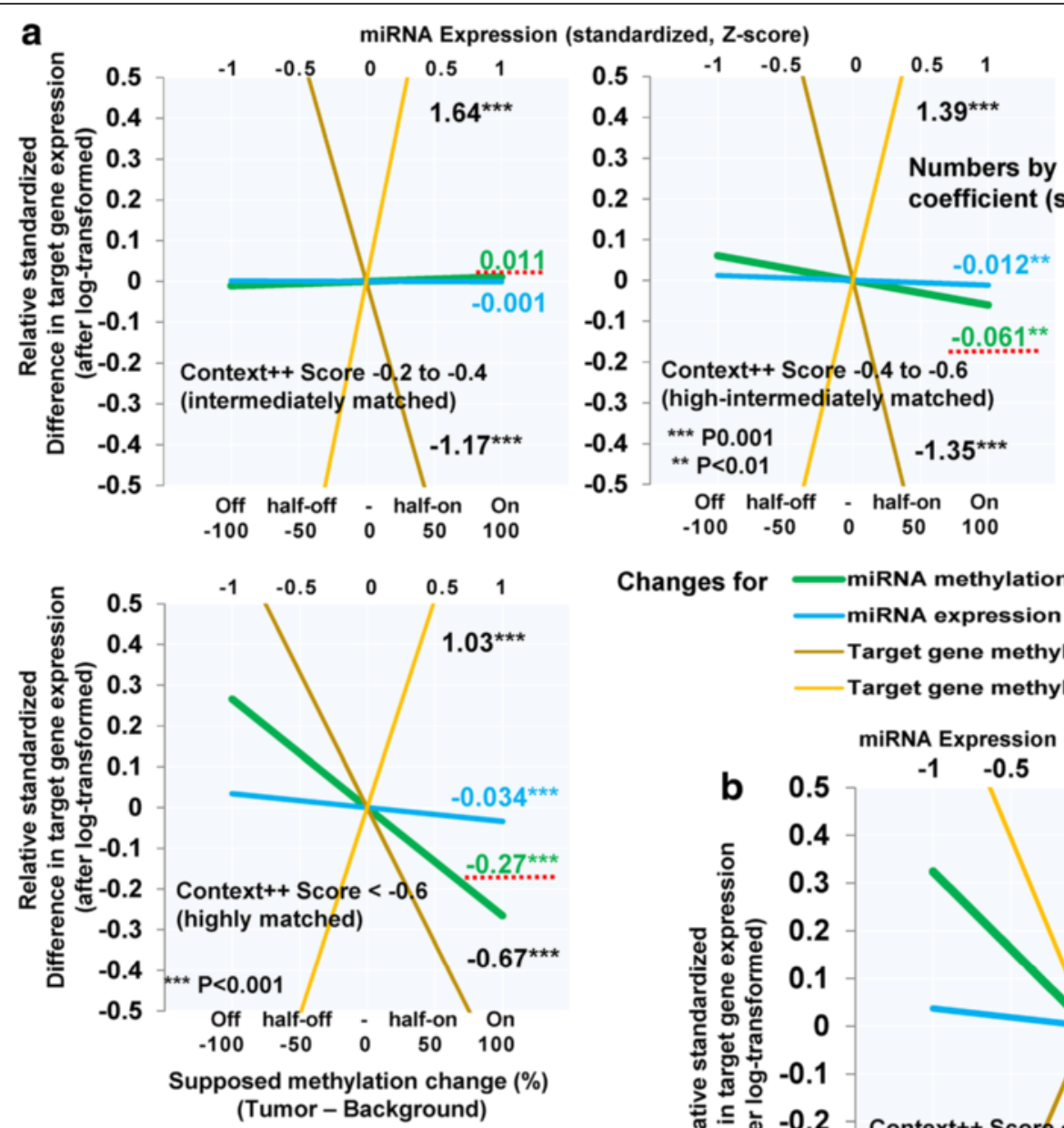

Changes for miRNA methylation

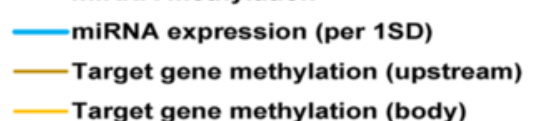

- Target gene methylation (body)
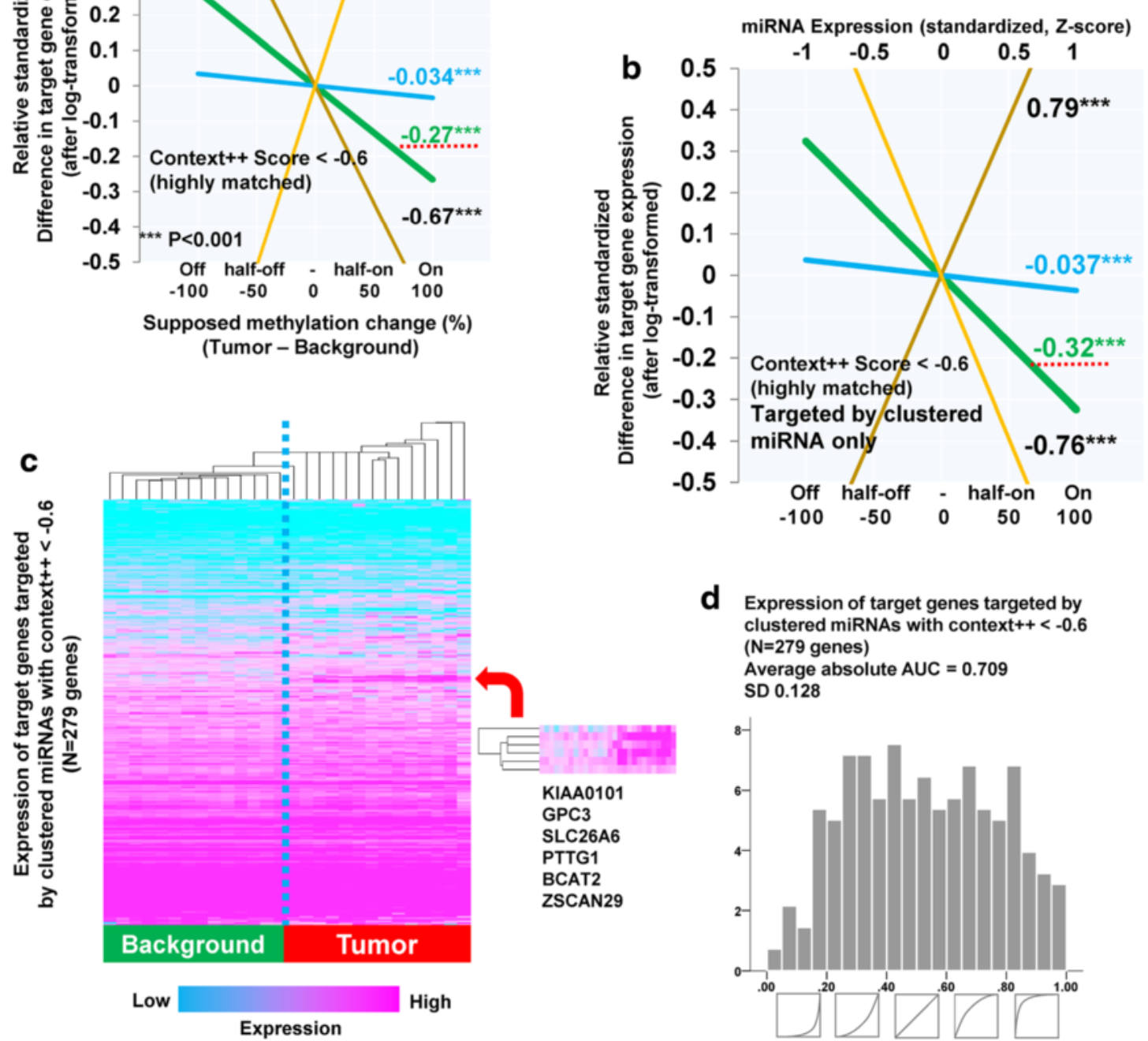

d Expression of target genes targeted by clustered miRNAs with context++ $<-0.6$ ( $N=279$ genes)

Average absolute AUC $=0.709$ SD 0.128

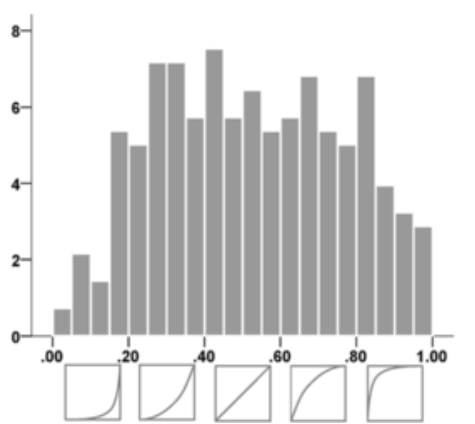

Fig. 6 (See legend on next page.) 


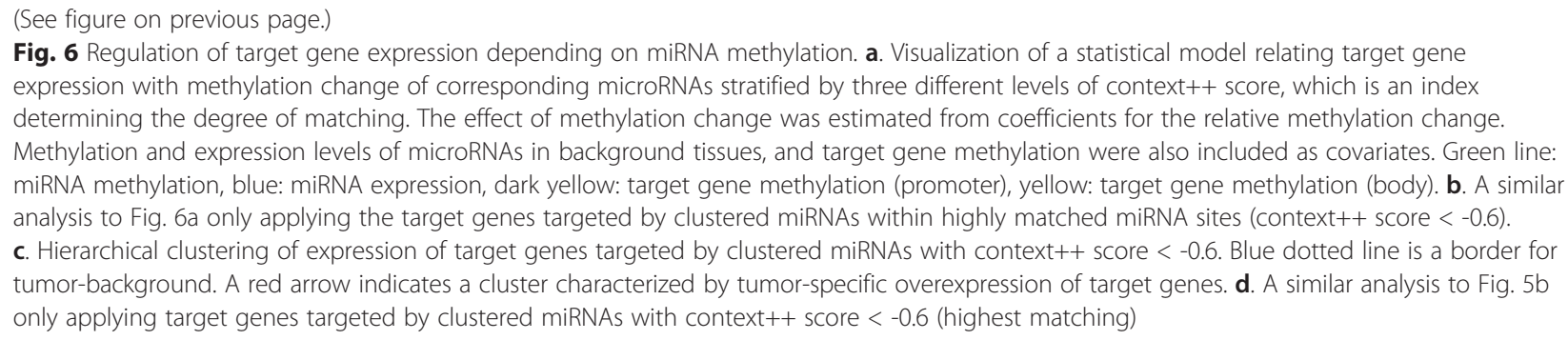

was found within both clusters, which is characterized by transcriptional regulation depending on methylation in promoter $\mathrm{CpG}$ islands. It also suggests that the difference in the transcriptional correlation of methylation change within upstream regions between each miRNAcoding region (please note it is different from "upstream region of the cluster") and protein-coding genes (defined as Fig. 1c, and compare Figs. 3a, 4 and $5 \mathrm{c}$ ) could be due to the difference in promoter activity of such regions. However, the correlations observed in the upstream regions of the clusters (see Fig. 4b, and c) are not necessarily representative pattern of miRNA transcription regulation (see the distribution of correlation coefficients in Fig. 4e). A similar inconsistent phenomenon was observed in the CTCF binding site. The CTCF binding site is considered important for miRNA expression regulation, as well as DNA methylation, and it modifies correlation patterns positively and negatively between expression and methylation $[18,30]$.

Although aberrant methylation within promoterbound CpG islands has been well studied for some time, the significance of gene body methylation and non-CpG island areas has until recently been minimized. Our results suggested the significance is not that small compared to promoter $\mathrm{CpG}$ islands, being consistent with recent reports about positive correlation between gene body methylation and expression [19, 31]. In tumor samples, large portions of gene bodies were relatively highly hypomethylated, and the relationship between this and tumor development should be studied. more Yang X et. al. described that gene body methylation may lead to genome instability and reduced efficiencies of posttranscriptional activity; other reports also support this idea [15]. Similar to miRNA regulation, direction of correlation between methylation of gene body and expression varies across the genome [13, 31].

In Fig. 6, miRNA methylation seems to regulate target expression directly amongst highly matched miRNA sites as it were independent from miRNA expression. Such results may be due to instability of expression measurement especially for miRNA, and stability of methylation measurement. In addition, transcription is regulated by multiple factors and dynamically changes moment by moment, with miRNAs also affecting surrounding/other cells by forming exosomes [32]. These findings might provide some explanation towards the discriminatory capability of tumor discrimination being present when methylation is considered (Fig. 5b). Moreover, our results in Fig. 6 indicate that expression of target genes of highly-matched clustered miRNAs differs between tumor and background tissues. This could be attributable to drastic hypomethylation of miRNA clusters. In general, global hypomethylation develops gradually through tumor development. For example, the pre-myeloma condition MGUS (Monoclonal gammopathy of undetermined significance) demonstrated intermediate methylation levels between normal and myeloma cells [15]. Pre-cancerous infections (e.g. HBV, HCV) also affect global methylation level in a similar way $[10,12]$. In addition, an established oncogene of hepatocellular carcinoma, GPC3 [33], was included in these target genes (Fig. 6c), and showed a tumor-specific overexpression pattern. Taken together, we consider that broad hypomethylation of miRNA clusters develops gradually through tumorigenesis, and not only as a specific phenotype but also a functionally significant factor.

\section{Conclusion}

We observed a dramatic negative shift in methylation levels within miRNA cluster regions, and investigated ways to correlate with expression data. Methylation changes in miRNAs were more indicative for target gene expression than miRNA expression change itself, suggesting the importance of genome-wide miRNA methylation for cancer development. Our study dynamically summarized the global miRNA hypomethylation and its genome-wide consequence in NBNC-HCC.

\section{Methods}

\section{Subjects and sample collection}

All subjects $(N=43)$ were diagnosed as HCC without HBV or HCV infection by expert hepatologists from Jan. 2004 to Dec. 2013. After informed consent for research was given, histologically diagnosed paired tissue samples (HCC and background liver) were collected from surgery samples at Osaka City University Hospital, Kyushu University Hospital, Tokushima University Hospital, and Tohoku University Hospital. This study was firstly 
approved by Nagoya City University ethical board as the primary center of the study (the submission number: 805-2), followed by ethical boards in each of the participating hospitals.

\section{Comprehensive analyses for DNA methylation and miRNA/mRNA expression}

The DNeasy Blood \& Tissue Kit (QIAGEN, USA) was used for DNA extraction, and mirVana miRNA Isolation Kit (Ambion, USA) and miRNeasy Mini Kit (QIAGEN, USA) was used for RNA extraction (the former was used for miRNA extraction, the latter was for total mRNA extraction). All collected samples were large enough to avoid major contamination of background tissue into tumor samples (median major axis: $47 \mathrm{~mm}$, interquartile range: $29-67.5 \mathrm{~mm}$, range: 16 to $170 \mathrm{~mm}$ ). Genome-wide DNA methylation status was assessed by an Infinium HumanMethylation450 BeadChip Kit (Illumina, USA, $N=43$ pairs of tumor and background). Genomewide mRNA expression analysis was performed using a SurePrint G3 Human GE 8x60K c2 Microarray (Agilent, USA, $N=15$ pairs of tumor and background). These analyses were performed at the CDM center, Takara Bio Inc. (Shiga, Japan). A 3D-Gene ${ }^{\circ}$ Human miRNA Oligo Chip V20 (Toray, Japan, $N=24$ pairs of tumor and background) was used for comprehensive miRNA expression analysis. Data files for these genome-wide experiments were submitted to ArrayExpress (https://www.ebi.ac.uk/ arrayexpress/) under accession numbers of E-MTAB4169, E-MTAB-4171, and E-MTAB-4170, respectively. All analyses were performed according to the procedure provided by the manufacturer. Target genes for miRNAs were identified using TargetScan 7.0 (http://www.targets can.org). We used all conserved miRNAs for human transcripts with greater context++ score (less than -0.2) [22].

\section{Statistical analyses}

General data mining and statistical analyses were performed via use of SPSS 20 (IBM, USA). Genome-wide methylation analysis was performed using beta values for all CpG loci represented on the Infinium HumanMethylation450BeadChip. For integrated analyses, the average methylation level or each gene (also stratified by upstream region/gene body and CpG island/shore/shelf/ non-island) was calculated, and then average expression of each gene (log-transformed signal and log-ratio) and average expression of each miRNA were annotated according to the corresponding methylation data. Clustering was performed using Cluster 3 [34]. Values measured by comprehensive analyses were transformed logarithmically and z-standardized as necessary. Continuous variables were generally compared by t-test or paired t-test. Area under curve (AUC) was calculated by receiver operating characteristic (ROC) analysis. General linear and liner mixed models were used for statistical modeling for determining expression-methylation association. In the mixed models, a patient identifier was included as a random effect. The other variables indicated in figures and tables were included as fixed effects. $P<0.05$ was considered significant.

\section{Availability of supporting data}

The data sets supporting the results of this article are available in the ArrayExpress repository, under accession numbers of E-MTAB-4169, E-MTAB-4170, and E-MTAB4171 in https://www.ebi.ac.uk/arrayexpress/.

\section{Additional files}

Additional file 1: Table S1. A general linear model for average methylation change attributed to genomic annotations of the probes. Table S2. A linear mixed model for miRNA expression change between tumor and background tissues attributed to their methylation change stratified by distance from CpG island and transcription start site. Table S3. Summary for methylation and expression of miRNA clusters. (Expression difference was z-transformed after obtaining log-ratio between background and tumor tissues. Thus, it was not equal to simple subtraction of background from tumor). Table S4. A linear mixed model for miRNA cluster expression (average within each cluster) change attributed to average cluster methylation change. Table S5. A linear mixed model for expression change of general proteincoding genes between tumor and background tissues attributed to their methylation change stratified by distance from CpG island and transcription start site. Table S6. A linear mixed model for target gene expression change between tumor and background tissues attributed to corresponding miRNA methylation change. (DOC $252 \mathrm{~kb}$ )

Additional file 2: Figure S1. Correlation coefficients between methylation/expression change of microRNAs within selected microRNA clusters (1p36.31, 1q24.3, 2p16.1, and 7q32.2). The selected clusters consist of the most and $2^{\text {nd }}$ most up-/down- regulated clusters (see Supplementary Table S3). (TIFF 3351 kb)

Additional file 3: Figure S2. Association between methylation levels of microRNA-coding regions and microRNA expression. A. Scatter plots showing correlation between clustered microRNA expression and methylation stratified by background and tumor tissues. B. Chromosome-wide correlation coefficients between methylation level as determined by each probe and corresponding microRNA expression. (TIFF $2122 \mathrm{~kb}$ )

\section{Abbreviations}

AUC: Area under curve; HCC: Hepatocellular carcinoma; HBV: Hepatitis B virus; HCV: Hepatitis C virus; NAFLD: Non-alcoholic fatty liver disease; NBNC-HCC: Non B non C hepatocellular carcinoma; miRNA: microRNA; mRNA: messenger RNA; SD: Standard deviation; ROC: Receiver operating characteristics.

\section{Competing interests}

The authors declare that they have no competing interests.

\section{Authors' contributions}

MN: Writing the manuscript, data analysis and interpretation. TM: Sample management and clinical data correction. AT, SK, KS, KK, MS, TU, and YK: Providing samples and clinical information, and advisor. El: Clinical data management. YN: Sample preparation and experiments. TO: Supervising technical and molecular biological considerations. YT: Conduct and supervising the overall research. All authors read and approved the final manuscript. 


\section{Acknowledgements}

This study was supported by Grants-in-Aid from the Ministry of Health, Labour and Welfare of Japan, and Japanese Agency for Medical Research and Development (Yasuhito Tanaka, Research ID: H25-kanen-ippan-005, and 15fk0210018h0003).

\section{Author details}

'Institute of Medical Science Hospital, Center for Translational Research, the University of Tokyo, Tokyo, Japan. ${ }^{2}$ Department of Gastroenterology, Teine Keijinkai Hospital, Sapporo, Japan. ${ }^{3}$ Department of Virology and Liver Unit, Nagoya City University Graduate School of Medical Sciences, Nagoya, Japan. ${ }^{4}$ Department of Hepatology, Osaka City University Graduate School of Medicine, Osaka, Japan. ${ }^{5}$ Department of Hepato-Biliary-Pancreatic Surgery, Osaka City University Graduate School of Medicine, Osaka, Japan. ${ }^{6}$ Department of Surgery and Science, Graduate School of Medical Sciences, Kyushu University, Fukuoka, Japan. 'Department of Surgery, Tokushima University, Tokushima, Japan. ${ }^{8}$ Division of Gastroenterology, Tohoku University Hospital, Sendai, Japan. ${ }^{9}$ Division of Molecular and Cellular Medicine, National Cancer Center Research Institute, Tokyo, Japan.

\section{Received: 10 February 2016 Accepted: 22 April 2016}

\section{Published online: 30 April 2016}

\section{References}

1. Ferlay J, Soerjomataram I, Dikshit R, Eser S, Mathers C, Rebelo M, et al. Cancer incidence and mortality worldwide: sources, methods and major patterns in GLOBOCAN 2012. Int J Cancer. 2015;136:E359-86.

2. Nishikawa H, Osaki Y. Non-B, non-C hepatocellular carcinoma (Review). Int J Oncol. 2013;43:1333-42.

3. Nagaoki Y, Hyogo H, Aikata H, Tanaka M, Naeshiro N, Nakahara T, et al. Recent trend of clinical features in patients with hepatocellular carcinoma. Hepatol Res. 2012;42:368-75.

4. Tokushige K, Hashimoto E, Horie Y, Taniai M, Higuchi S. Hepatocellular carcinoma in Japanese patients with nonalcoholic fatty liver disease, alcoholic liver disease, and chronic liver disease of unknown etiology: report of the nationwide survey. J Gastroenterol. 2011;46:1230-7.

5. de Martel C, Maucort-Boulch D, Plummer M, Franceschi S. World-wide relative contribution of hepatitis $B$ and $C$ viruses in hepatocellular carcinoma. Hepatology. 2015;62:1190-200.

6. Nojima M, Suzuki H, Toyota M, Watanabe Y, Maruyama R, Sasaki S, et al. Frequent epigenetic inactivation of SFRP genes and constitutive activation of Wnt signaling in gastric cancer. Oncogene. 2007;26:4699-713.

7. Nojima M, Maruyama R, Yasui H, Suzuki H, Maruyama Y, Tarasawa I, et al. Genomic screening for genes silenced by DNA methylation revealed an association between RASD1 inactivation and dexamethasone resistance in multiple myeloma. Clin Cancer Res. 2009;15:4356-64.

8. Villanueva A, Portela A, Sayols S, Battiston C, Hoshida Y, Mendez-Gonzalez J, et al. DNA methylation-based prognosis and epidrivers in hepatocellular carcinoma. Hepatology. 2015;61:1945-56.

9. Shen J, Wang S, Zhang YJ, Wu HC, Kibriya MG, Jasmine F, et al. Exploring genome-wide DNA methylation profiles altered in hepatocellular carcinoma using Infinium HumanMethylation 450 BeadChips. Epigenetics. 2013;8:34-43.

10. Okamoto Y, Shinjo K, Shimizu Y, Sano T, Yamao K, Gao W, et al. Hepatitis virus infection affects DNA methylation in mice with humanized livers. Gastroenterology. 2014;146:562-72.

11. Shen J, Wang S, Zhang YJ, Kappil MA, Chen Wu H, Kibriya MG, et al. Genome-wide aberrant DNA methylation of microRNA host genes in hepatocellular carcinoma. Epigenetics. 2012;7:1230-7.

12. Lee SM, Lee YG, Bae JB, Choi JK, Tayama C, Hata K, et al. HBx induces hypomethylation of distal intragenic $\mathrm{CpG}$ islands required for active expression of developmental regulators. Proc Natl Acad Sci U S A. 2014; 111:9555-60.

13. Kulis M, Heath S, Bibikova M, Queiros AC, Navarro A, Clot G, et al. Epigenomic analysis detects widespread gene-body DNA hypomethylation in chronic lymphocytic leukemia. Nat Genet. 2012;44:1236-42.

14. Feinberg AP, Vogelstein B. Hypomethylation distinguishes genes of some human cancers from their normal counterparts. Nature. 1983;301: 89-92.

15. Aoki Y, Nojima M, Suzuki H, Yasui H, Maruyama R, Yamamoto E, et al. Genomic vulnerability to LINE-1 hypomethylation is a potential determinant of the clinicogenetic features of multiple myeloma. Genome Med. 2012;4:101.

16. Anwar SL, Lehmann U. DNA methylation, microRNAs, and their crosstalk as potential biomarkers in hepatocellular carcinoma. World J Gastroenterol. 2014;20:7894-913

17. Xia H, Ooi LL, Hui KM. MicroRNA-216a/217-induced epithelial-mesenchymal transition targets PTEN and SMAD7 to promote drug resistance and recurrence of liver cancer. Hepatology. 2013;58:629-41.

18. Manodoro F, Marzec J, Chaplin T, Miraki-Moud F, Moravcsik E, Jovanovic JV, et al. Loss of imprinting at the 14q32 domain is associated with microRNA overexpression in acute promyelocytic leukemia. Blood. 2014;123:2066-74.

19. Yang X, Han H, De Carvalho DD, Lay FD, Jones PA, Liang G. Gene body methylation can alter gene expression and is a therapeutic target in cancer. Cancer Cell. 2014;26:577-90.

20. Attema JL, Bert AG, Lim YY, Kolesnikoff N, Lawrence DM, Pillman KA, et al. Identification of an enhancer that increases miR-200b 200a 429 gene expression in breast cancer cells. PLoS One. 2013;8, e75517.

21. Li P, Sheng C, Huang L, Zhang H, Huang L, Cheng Z, et al. MiR-183/-96/-182 cluster is up-regulated in most breast cancers and increases cell proliferation and migration. Breast Cancer Res. 2014;16:473.

22. Agarwal V, Bell GW, Nam JW, Bartel DP. Predicting effective microRNA target sites in mammalian mRNAs. Elife. 2015;4:e05005.

23. Vaira V, Elli F, Forno I, Guarnieri V, Verdelli C, Ferrero S, et al. The microRNA cluster C19MC is deregulated in parathyroid tumours. J Mol Endocrinol. 2012:49:115-24.

24. Fornari F, Milazzo M, Chieco P, Negrini M, Marasco E, Capranico G, et al. In hepatocellular carcinoma miR-519d is up-regulated by p53 and DNA hypomethylation and targets CDKN1A/p21, PTEN, AKT3 and TIMP2. J Pathol. 2012;227:275-85.

25. Baer C, Claus R, Frenzel LP, Zucknick M, Park YJ, Gu L, et al. Extensive promoter DNA hypermethylation and hypomethylation is associated with aberrant microRNA expression in chronic lymphocytic leukemia. Cancer Res. 2012;72:3775-85.

26. Berenstein R, Blau O, Nogai A, Waechter M, Slonova E, Schmidt-Hieber M, et al. Multiple myeloma cells alter the senescence phenotype of bone marrow mesenchymal stromal cells under participation of the DLK1-DIO3 genomic region. BMC Cancer. 2015;15:68.

27. Jeong M, Sun D, Luo M, Huang Y, Challen GA, Rodriguez B, et al. Large conserved domains of low DNA methylation maintained by Dnmt3a. Nat Genet. 2014;46:17-23.

28. Ito S, Shen L, Dai Q, Wu SC, Collins LB, Swenberg JA, et al. Tet proteins can convert 5-methylcytosine to 5-formylcytosine and 5-carboxylcytosine. Science. 2011;333:1300-3.

29. Torres-Martin M, Lassaletta L, de Campos JM, Isla A, Pinto GR, Burbano RR, et al. Genome-wide methylation analysis in vestibular schwannomas shows putative mechanisms of gene expression modulation and global hypomethylation at the HOX gene cluster. Genes Chromosomes Cancer. 2015:54:197-209.

30. Saito $Y$, Saito H. Role of CTCF in the regulation of microRNA expression. Front Genet. 2012;3:186.

31. Yu B, Russanova VR, Gravina S, Hartley S, Mullikin JC, Ignezweski A, et al. DNA methylome and transcriptome sequencing in human ovarian granulosa cells links age-related changes in gene expression to gene body methylation and 3'-end GC density. Oncotarget. 2015;6:3627-43.

32. Bullerdiek J, Flor I. Exosome-delivered microRNAs of "chromosome 19 microRNA cluster" as immunomodulators in pregnancy and tumorigenesis. Mol Cytogenet. 2012;5:27.

33. Dargel C, Bassani-Sternberg M, Hasreiter J, Zani F, Bockmann JH, Thiele F, et al. T Cells Engineered to Express a T-Cell Receptor Specific for Glypican-3 to Recognize and Kill Hepatoma Cells In Vitro and in Mice. Gastroenterology. 2015;149:1042-52

34. de Hoon MJ, Imoto S, Nolan J, Miyano S. Open source clustering software. Bioinformatics. 2004;20:1453-4. 